\title{
Immune Checkpoint Inhibitors in Pediatric Solid Tumors: Status in 2018
}

\author{
Tanvir F. Kabir, BA, ${ }^{1}$ Aman Chauhan, MD, ${ }^{2}$ Lowell Anthony, MD, ${ }^{2}$ Gerhard C. Hildebrandt, MD $^{3}$ \\ ${ }^{1}$ University of Kentucky College of Medicine, Lexington, KY ${ }^{2}$ Division of Medical Oncology, Markey Cancer Center, University of Kentucky, \\ Lexington, $\mathrm{KY}{ }^{3}$ Division of Bone Marrow Transplant and Benign Hematology, University of Kentucky, Lexington, KY
}

Background: Checkpoint inhibitors have transformed the treatment of cancer in adults. This class of drugs has demonstrated encouraging results in various malignancies such as metastatic melanoma, bladder cancer, renal cancer, and non-small cell lung carcinoma. However, researchers have only begun investigating the effectiveness and tolerability of checkpoint inhibitors in pediatric patients.

Methods: We conducted a review of PubMed indexed literature and clinicaltrials.gov using combinations of the keywords checkpoint, inhibitor, pediatric, CTLA-4 (cytotoxic T lymphocyte antigen-4), PD-1 (programmed cell death-1), and PD-L1 (programmed cell death receptor-1 ligand) to find every recently completed and ongoing trial evaluating checkpoint inhibitors in patients younger than 21 years old. Pertinent articles and clinical trials discussing the role of immune checkpoint inhibitors in the pediatric population were selected for final analysis and manuscript citation.

Results: This review presents an overview of the cellular mechanisms involved in checkpoint inhibition and of studies evaluating checkpoint inhibitors in humans. The review also details results and side effects from studies conducted with pediatric patients, current pediatric clinical trials, and future implications.

Conclusion: Immune checkpoint inhibitors have the potential to further therapeutic advances in pediatric oncology; however, we need more clinical trials and combination drug strategies targeted toward pediatric cancers.

Keywords: Costimulatory and inhibitory T-cell receptors, CTLA-4 antigen, immune checkpoint inhibitors, immunity-cellular, immunotherapy, programmed cell death 1 receptor

Address correspondence to Aman Chauhan, MD, Division of Medical Oncology, Markey Cancer Center, 800 Rose St., Lexington, KY 40536-0293. Tel: (859) 323-6522. Email: amanchauhan@uky.edu

\section{INTRODUCTION}

Checkpoint inhibitors are among the most researched anticancer agents of the $21^{\text {st }}$ century. Rarely do scientists find a new class of drugs that demonstrates both efficacy as well as tolerable toxicity in a multitude of different cancer types. This group of drugs targets signaling pathways usually used to modulate host immune responses as part of the normal regulation of immunity and establishment of tolerance. However, these immune checkpoints can be hijacked by cancer cells to silence the host's immune response. Multiple pathways have been discovered through which malignant cells can deactivate immune cells-including $\mathrm{T}$ and $\mathrm{B}$ lymphocytesfrom targeting these cells. During cancer progression, $\mathrm{T}$ cells become desensitized and are unable to target malignant cells. ${ }^{1,2}$ This desensitization, also known as exhaustion, can occur when tumor cells upregulate inhibitory receptors such as programmed cell death receptor-1 ligand (PD-L1) and cytotoxic T lymphocyte antigen-4 (CTLA-4). ${ }^{3}$ Checkpoint inhibitors seek to reverse this inhibition so that an immune response can be mounted against the malignant cells.
We conducted a review of PubMed indexed literature and clinicaltrials.gov using combinations of the keywords checkpoint, inhibitor, pediatric, CTLA-4, PD-1 (programmed cell death-1), and PD-L1 to find every recently completed and ongoing trial evaluating checkpoint inhibitors in patients younger than 21 years old. Given that most clinical trials to date have evaluated checkpoint inhibitors in adult patients, this review presents a brief summary of studies in humans of all age groups and then focuses on results and side effects in pediatric patients. This review also presents future directions and current clinical trials evaluating checkpoint inhibitors in patients $<21$ years.

\section{CELLULAR MECHANISMS INVOLVED IN CHECKPOINT INHIBITION}

Ishida et al first reported the PD-1 pathway in $1992 .{ }^{4} \mathrm{PD}-1$ is a transmembrane glycoprotein made up of an immunoglobulin domain, intracellular domain, transmembrane, and stalk comprised of 20 amino acids. ${ }^{5,6}$ This glycoprotein is an immunoreceptor within the CD28/CTLA-4 class of 
T lymphocyte regulators. ${ }^{7,8}$ The protein is structurally similar to antigen receptors and is considered an intermediate between CD28 and antigen receptors (Figure). ${ }^{5}$

PD-1 acts as a negative regulator that inhibits $T$ cell activity by interacting with either of two ligands located on normal healthy tissue and tumor cells, PD-L1 or PD-L2. ${ }^{9,10}$ When the PD-L1 ligand located on normal or tumor cells interacts with the PD-1 cell receptor on an immune cell, the immune cell's activity is suppressed. ${ }^{11}$ On the molecular level, the interaction leads to the phosphorylation of intracellular residues which recruit SHP-2 that dephosphorylates downstream signaling molecules such as ZAP70. This process attenuates the $T$ cell receptor (TCR)/CD28 signal on $T$ cells. ${ }^{12,13}$ The PD-1 pathway also deactivates $B$ cells, and blockade of the pathway has been shown to increase proliferation and activation of B cells. ${ }^{14}$ In fact, PD-1 can be expressed on B cells, CD8+ and CD4+ T cells, dendritic cells, natural killer cells, and monocytes. ${ }^{8,15,16}$

PD-L1 and PD-L2 ligands are structurally similar. ${ }^{8}$ Both PD-L1 and PD-L2 ligands can suppress T cell activity, but they differ in their distribution throughout the body. PD-L2 has a more limited distribution, including macrophages and dendritic cells. PD-L1, on the other hand, is found in many more tissues. The mRNA of PD-L1 is in almost all human tissues in addition to various malignant cells, possibly a mechanism for the immune system to recognize every cell in the body. ${ }^{9,17,18}$

CTLA-4 is another pathway that tumors can exploit to attenuate $T$ cell activity but through a different mechanism than PD-1. ${ }^{7}$ Brunet et al discovered the CTLA-4 pathway in

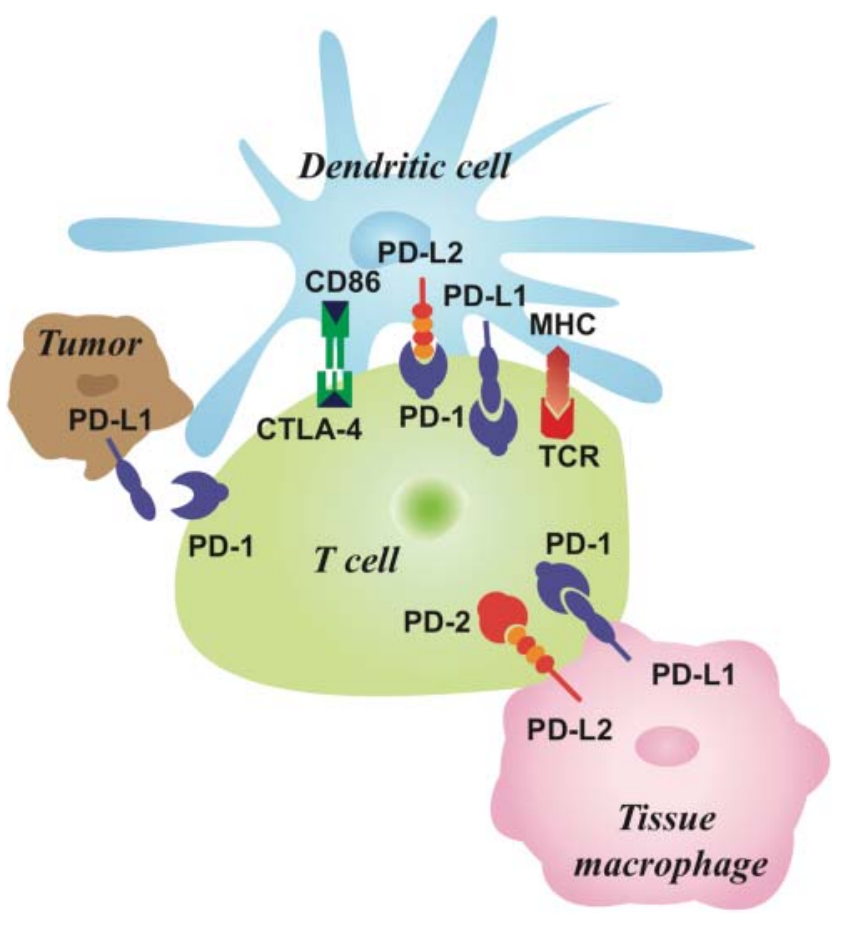

Figure. Distribution of immune checkpoint in tumor cells and immune microenvironment. CTLA-4, cytotoxic T lymphocyte antigen-4; MHC, major histocompatibility complex; PD-1/ PD-2, programmed cell death-1/-2; PD-LI/PD-L2, programmed cell death receptor-1/-2 ligand; TCR, T cell receptor.
1987. ${ }^{19}$ TCR ligation results in CTLA-4 receptor upregulation that competes with CD28 in binding to the B7 ligand. This reduced costimulation via CD28 results in decreased T lymphocyte activity. ${ }^{20,21}$

In adult human clinical trials, the blockade of these pathways with checkpoint inhibitors such as ipilimumab (targeting CTLA-4) and nivolumab (targeting PD-1) has demonstrated encouraging results, although not in all cancer types. The drug ipilimumab was the first immune checkpoint inhibitor against CTLA-4 to increase survival in adults with metastatic melanoma. ${ }^{22,23}$ The fully human monoclonal antibody tremelimumab (targeting CTLA-4) has demonstrated success against various cancers including melanoma. ${ }^{24}$ The checkpoint inhibitor nivolumab (targeting PD-1) demonstrated successful results against melanoma, renal cell carcinoma, and non-small cell lung cancer.,25 Pembrolizumab (targeting PD-1) showed positive results against metastatic non-small cell lung cancer, extending overall survival with fewer side effects compared to standard of care platinum-based chemotherapy. ${ }^{26}$ Another PD-1 inhibitor, atezolizumab, has been approved as second-line treatment for non-small cell lung cancer. $^{27}$

Furthermore, the combination of PD-1 and CTLA-4 blockage shows higher response rates against metastatic melanoma compared to each agent alone. ${ }^{28}$ The benefits of checkpoint inhibitors are not restricted to solid tumors. Significant activity has been seen in hematologic malignancies as well. Use of nivolumab or pembrolizumab, both targeting the PD-1/PD-L1 ligand, has resulted in significant responses in relapsed/refractory patients with Hodgkin lymphoma with respect to both cytoreduction and duration of response. ${ }^{29-31}$ Early data suggest activity in non-Hodgkin lymphomas as well. $^{32,33}$

\section{CHECKPOINT INHIBITORS IN PEDIATRICS}

Immune checkpoint drug development focused on adult cancers in the past; however, there has been a push to evaluate this class of therapeutics specifically in pediatric cancers. While clinical trials testing checkpoint inhibitors in pediatric patients have been limited, some results have been promising. Pembrolizumab has received US Food and Drug Administration approval for treatment of Hodgkin lymphoma in pediatric patients, the first such approval for the pediatric population. ${ }^{34}$

PD-L1 is known to be expressed in common pediatric cancers. ${ }^{1,6,35}$ Karim et al evaluated PD-L1 expression in various hematologic malignancies and found high expression of PDL1 in $100 \%$ of Hodgkin lymphoma cases (10/10). ${ }^{36}$ They also discovered PD-L1 expression in $80 \%$ of diffuse large B cell lymphoma (DLBCL) cases (8/10) and high mutational burden in $59 \%$ of 253 DLBCL cases. Acute myeloid leukemia and acute lymphoblastic leukemia had significantly less PDL1 expression: $24 \%$ and $38 \%$ of sample cases, respectively. In other studies, $65 \%-87 \%$ of pediatric patients with Hodgkin lymphoma demonstrated PD-L1 upregulation, and a higher level correlated with poor survival. ${ }^{37-41}$ Researchers have also demonstrated that $75 \%-100 \%$ of gliomas in a sample of cases exhibited PD-L1 expression, which correlated with the severity of disease. .2,43 $^{4}$

However, several challenges exist before checkpoint inhibition becomes more widespread in the treatment of pediatric cancers. PD-L1 and PD-L2 expression has been 
found to be relatively low in many pediatric solid tumors. ${ }^{44}$ Furthermore, the checkpoint inhibition-induced augmented immune response is associated with a distinct pattern of side effects and toxicities, also known as immune-related adverse events (irAEs).

A number of published studies describe the efficacy and side effect profile of checkpoint inhibitors in pediatric patients. ${ }^{45}$ In a phase 1 trial, ipilimumab was studied in 33 patients between the ages of 28 months and 21 years with advanced solid malignancies including melanoma, bladder, renal, neuroblastoma, and sarcoma cancers. ${ }^{46}$ Fifty-five percent of the patients developed a side effect, with $27 \%$ developing a severe side effect (grade 3 or 4 irAE). Side effects were similar to what has been seen in adult patients such as pneumonitis, endocrinopathies, and colitis. However, survival increased in patients who experienced side effects. Six of the 33 patients exhibited stable disease for at least 4 to 10 cycles.

In another study, an 8-year-old child with advanced melanoma demonstrated positive results after treatment with ipilimumab at $3 \mathrm{mg} / \mathrm{kg}$. ${ }^{47}$ The patient experienced thyroiditis and an infusion reaction, but the infusion reaction only occurred after the first course of treatment. Steroids and antihistamines prevented further reactions.

In various other case reports, 5 pediatric patients aged 322 years with advanced glioblastoma and Hodgkin lymphoma received nivolumab. The 2 patients with Hodgkin lymphoma demonstrated a long-term response, while 2 of the children with glioblastoma demonstrated controlled disease for more than 1 year. ${ }^{34,48,49}$ The fifth child, a 10-year-old with glioblastoma, experienced malignant cerebral edema and uncal herniation after treatment with nivolumab, possibly secondary to an inflammatory response. ${ }^{50}$

Finally, 5 pediatric patients aged 3-7 years with medulloblastoma, glioblastoma, diffuse intrinsic pontine glioma, and teratoid-rhabdoid tumor were treated with pembrolizumab. Every patient progressed; median survival was 3.2 months. ${ }^{51}$ Side effects were mild and included diarrhea, rash, and transaminitis.

Theoretically, immune checkpoint inhibition may strengthen graft-versus-tumor response in patients with pediatric cancers who require allogeneic hematopoietic stem cell transplantation (HSCT) for relapse, but it also carries the risk of eliciting severe graft-versus-host disease (GVHD). ${ }^{45,52,53}$ Haverkos et al conducted a retrospective analysis of 31 patients with lymphoma who received anti-PD-1 monoclonal antibodies after allogeneic $\mathrm{HSCT}^{54}$ Fifty percent of the patients had a complete response, and $27 \%$ had a partial response. However, 55\% of the patients developed GVHD after treatment with the anti-PD-1 monoclonal antibodies, and $26 \%$ of the group died of complications from GVHD. The authors of this study concluded that PD-1 inhibition in allogeneic HSCT patients is effective but often complicated by severe GVHD; thus, PD-1 inhibitors cannot be recommended with HSCT until the side effects can be properly managed.

\section{FUTURE OF CHECKPOINT INHIBITORS}

Checkpoint inhibitors will likely not be used as monotherapy in the future, except for select biomarker-enriched populations, but rather will likely be used synergistically with other modalities. ${ }^{1}$ The combination of different checkpoint inhibitors has shown great promise in adult patients. Nivolu- mab (targeting PD-1) combined with ipilimumab (targeting CTLA-4) in metastatic melanoma resulted in $>80 \%$ tumor regression in $30 \%$ of adult patients. ${ }^{55}$ This combination also showed promising results in recurrent glioblastoma and advanced renal cell carcinoma. ${ }^{1}$ One case reports the complete remission of refractory squamous cell head and neck cancer in a 47-year-old patient after treatment with nivolumab combined with ipilimumab. ${ }^{56}$ Currently, clinical trials are evaluating combinations of checkpoint inhibitors in pediatric patients. A phase 2 clinical trial that began in June 2017 to evaluate nivolumab with or without ipilimumab is actively recruiting pediatric patients with high-grade primary central nervous system cancers (NCT03130959).

Chemotherapy combined with checkpoint inhibitors is also currently under investigation. Dacarbazine combined with ipilimumab increased survival in patients with advanced melanoma in comparison to dacarbazine alone. ${ }^{23} \mathrm{~A}$ trial that began in September 2018 is comparing nivolumab in combination with cyclophosphamide, vinblastine, and/or capecitabine in pediatric patients (NCT03585465). Researchers are also testing combinations of checkpoint inhibitors with targeted therapies including angiogenesis inhibitors, tyrosine kinase inhibitors, and other monoclonal antibodies. ${ }^{1}$ Ipilimumab combined with bevacizumab has shown success with mild side effects in metastatic melanoma. ${ }^{57,58} \mathrm{~A}$ phase I study of ipilimumab and imatinib in both children and adults that began in February 2013 is actively recruiting (NCT01738139). Finally, a study in France that began in August 2016 is evaluating nivolumab in combination with lirilumab, a human monoclonal antibody that binds to KIR2DL1/ 2L3 (a receptor expressed on natural killer cells and a minority of T cells that regulates function) (NCT02813135).

Numerous trials evaluating checkpoint inhibitors in all age groups are underway, but few focus strictly on pediatric cancers. However, 9 ongoing and 2 completed trials evaluating checkpoint inhibitors strictly in pediatric patients up to age 21 years are summarized in the Table. Two trials are testing ipilimumab alone, while 3 trials are evaluating nivolumab alone. One trial combines ipilimumab with nivolumab, and another trial combines nivolumab with lirilumab. Additionally, one trial is investigating nivolumab with cyclophosphamide, vinblastine, capecitabine, or a combination of these drugs. Three trials are evaluating other checkpoint inhibitors in pediatric cancers: pembrolizumab, durvalumab, and pidilizumab.

\section{CONCLUSION}

Modulation of the human immune system has transformed cancer therapy, and checkpoint inhibition is one of the many successful forms of immunotherapy used to treat malignancies. Given their tolerable side effect profile and potential for a durable response, checkpoint inhibitors will likely be used in conjunction with other modalities to extend life expectancy. Immune checkpoints have shown early signals of efficacy when combined with chemotherapy, targeted therapies, and other forms of immunotherapy. More clinical trials need to be performed to evaluate the effect of combination therapy in a wider range of cancer types, particularly in pediatric cancers. 


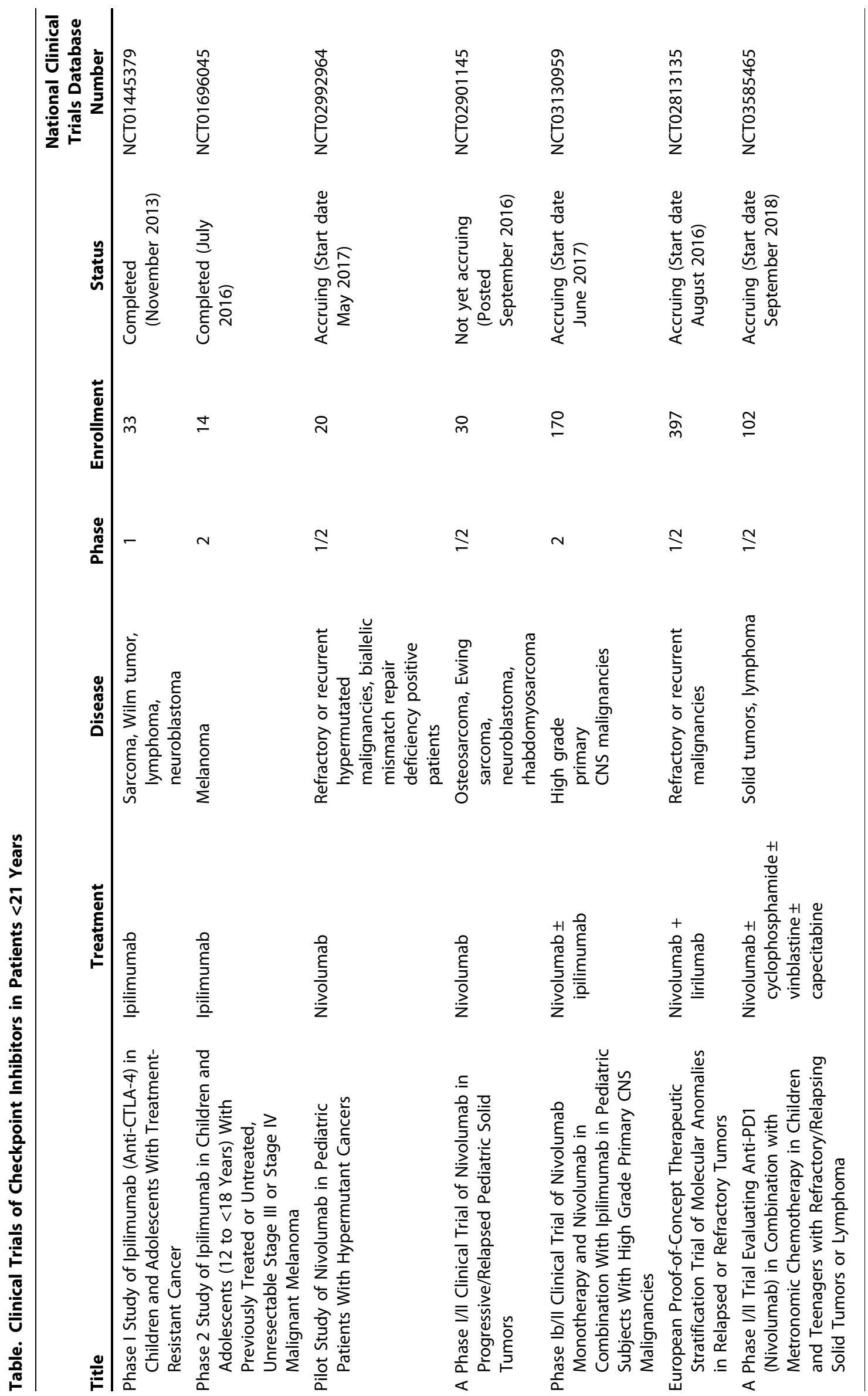




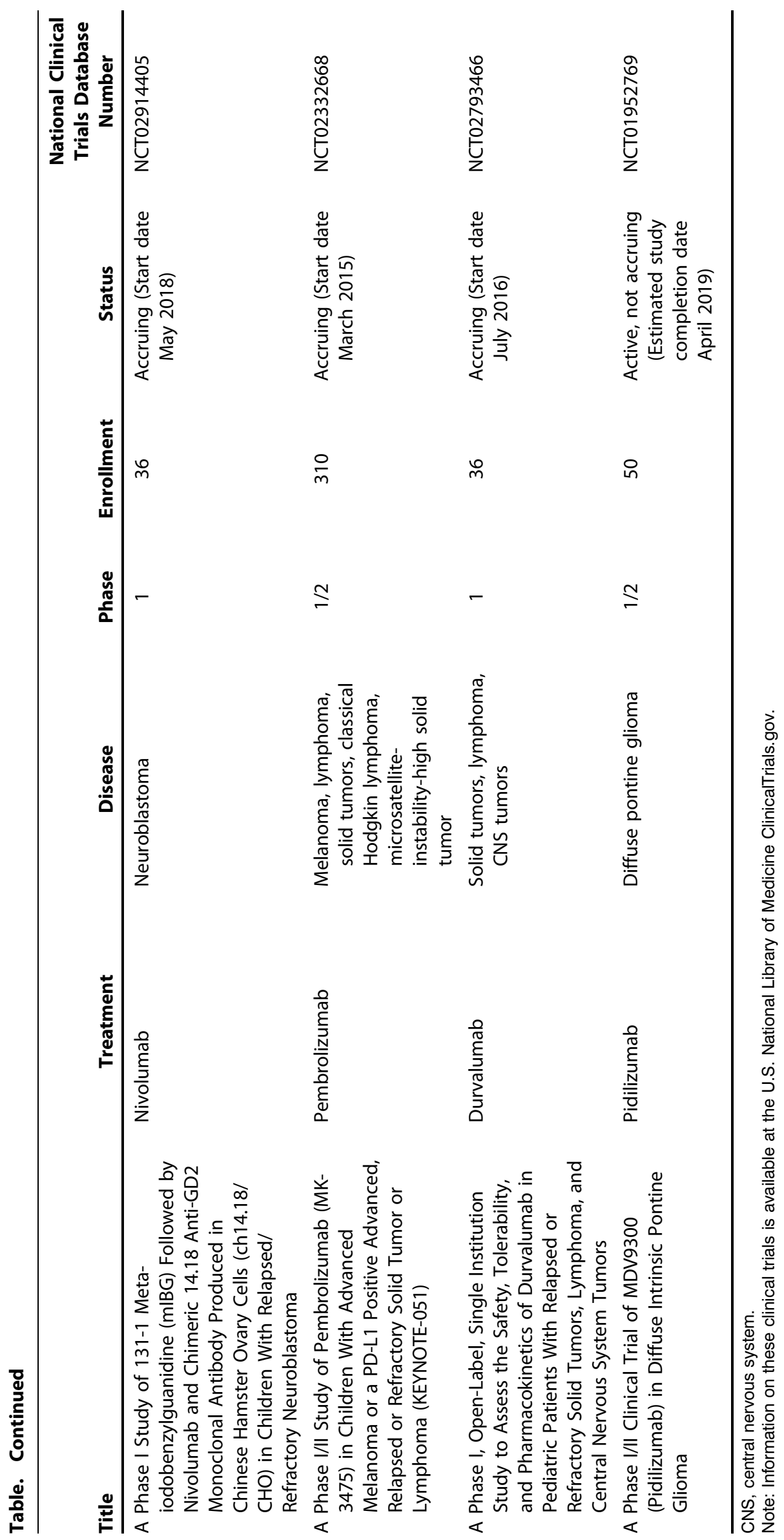




\section{ACKNOWLEDGMENTS}

The authors would like to thank Donna Gilbreath (Markey Cancer Center Research Communications Office) for her help with manuscript editing, formatting, and graphic design. The authors have no financial or proprietary interest in the subject matter of this article.

\section{REFERENCES}

1. Park JA, Cheung NV. Limitations and opportunities for immune checkpoint inhibitors in pediatric malignancies. Cancer Treat Rev. 2017 Jul;58:22-33. doi: 10.1016/j.ctrv.2017.05.006.

2. Peggs KS, Quezada SA, Allison JP. Cell intrinsic mechanisms of T-cell inhibition and application to cancer therapy. Immunol Rev. 2008 Aug;224:141-165. doi: 10.1111/j.1600065X.2008.00649.x.

3. Wherry EJ, Kurachi M. Molecular and cellular insights into T cell exhaustion. Nat Rev Immunol. 2015 Aug;15(8):486-499. doi: 10.1038/nri3862.

4. Ishida Y, Agata Y, Shibahara K, Honjo T. Induced expression of PD-1, a novel member of the immunoglobulin gene superfamily, upon programmed cell death. EMBO J. 1992 Nov; 11(11):3887-3895.

5. Cheng X, Veverka V, Radhakrishnan A, et al. Structure and interactions of the human programmed cell death 1 receptor. J Biol Chem. 2013 Apr 26;288(17):11771-11785. doi: 10.1074/jbc. M112.448126.

6. van Dam LS, de Zwart VM, Meyer-Wentrup FA. The role of programmed cell death-1 (PD-1) and its ligands in pediatric cancer. Pediatr Blood Cancer. 2015 Feb;62(2):190-197. doi: 10.1002/pbc.25284.

7. Pardoll DM. The blockade of immune checkpoints in cancer immunotherapy. Nat Rev Cancer. 2012 Mar 22;12(4):252-264. doi: $10.1038 / \mathrm{nrc3} 323$.

8. Keir ME, Butte MJ, Freeman GJ, Sharpe AH. PD-1 and its ligands in tolerance and immunity. Annu Rev Immunol. 2008;26:677704. doi: 10.1146/annurev.immunol.26.021607.090331.

9. Freeman GJ, Long AJ, Iwai Y, et al. Engagement of the PD-1 immunoinhibitory receptor by a novel B7 family member leads to negative regulation of lymphocyte activation. J Exp Med. 2000 Oct 2;192(7):1027-1034.

10. Latchman Y, Wood CR, Chernova T, et al. PD-L2 is a second ligand for PD-1 and inhibits T cell activation. Nat Immunol. 2001 Mar;2(3):261-268.

11. Dong H, Strome SE, Salomao DR, et al. Tumor-associated B7-H1 promotes T-cell apoptosis: a potential mechanism of immune evasion. Nat Med. 2002 Aug;8(8):793-800.

12. Chemnitz JM, Parry RV, Nichols KE, June CH, Riley JL. SHP-1 and SHP-2 associate with immunoreceptor tyrosine-based switch motif of programmed death 1 upon primary human $\mathrm{T}$ cell stimulation, but only receptor ligation prevents $T$ cell activation. J Immunol. 2004 Jul 15;173(2):945-954.

13. Sheppard KA, Fitz LJ, Lee JM, et al. PD-1 inhibits T-cell receptor induced phosphorylation of the ZAP70/CD3zeta signalosome and downstream signaling to PKCtheta. FEBS Lett. 2004 Sep 10;574(1-3):37-41.

14. Thibult ML, Mamessier E, Gertner-Dardenne J, et al. PD-1 is a novel regulator of human B-cell activation. Int Immunol. 2013 Feb;25(2):129-137. doi: 10.1093/intimm/dxs098.

15. Okazaki T, Chikuma S, Iwai Y, Fagarasan S, Honjo T. A rheostat for immune responses: the unique properties of PD- 1 and their advantages for clinical application. Nat Immunol. 2013 Dec;14 (12):1212-1218. doi: 10.1038/ni.2762.
16. Zou W, Chen L. Inhibitory B7-family molecules in the tumour microenvironment. Nat Rev Immunol. 2008 Jun;8(6):467-477. doi: $10.1038 /$ nri2326.

17. Dong H, Zhu G, Tamada K, Chen L. B7-H1, a third member of the B7 family, co-stimulates T-cell proliferation and interleukin10 secretion. Nat Med. 1999 Dec;5(12):1365-1369.

18. Brown JA, Dorfman DM, Ma FR, et al. Blockade of programmed death-1 ligands on dendritic cells enhances $T$ cell activation and cytokine production. J Immunol. 2003 Feb 1;170(3): 1257-1266.

19. Brunet JF, Denizot F, Luciani MF, et al. A new member of the immunoglobulin superfamily-CTLA-4. Nature. 1987;328 (6127):267-270.

20. Krummel MF, Allison JP. CD28 and CTLA-4 have opposing effects on the response of T cells to stimulation. J Exp Med. 1995 Aug 1;182(2):459-465.

21. Walunas TL, Lenschow DJ, Bakker CY, et al. CTLA-4 can function as a negative regulator of T cell activation. Immunity. 1994 Aug;1(5):405-413.

22. Hodi FS, O'Day SJ, McDermott DF, et al. Improved survival with ipilimumab in patients with metastatic melanoma. $N$ Engl $J$ Med. 2010 Aug 19;363(8):711-723. doi: 10.1056/ NEJMoa1003466.

23. Robert C, Thomas L, Bondarenko I, et al. Ipilimumab plus dacarbazine for previously untreated metastatic melanoma. N Engl J Med. 2011 Jun 30;364(26):2517-2526. doi: 10.1056/ NEJMoa1104621.

24. Comin-Anduix B, Escuin-Ordinas $\mathrm{H}$, Ibarrondo FJ. Tremelimumab: research and clinical development. Onco Targets Ther. 2016 Mar 23;9:1767-1776. doi: 10.2147/OTT. S65802.

25. Topalian SL, Hodi FS, Brahmer JR, et al. Safety, activity, and immune correlates of anti-PD-1 antibody in cancer. $N$ Engl $J$ Med. 2012 Jun 28;366(26):2443-2454. doi: 10.1056/ NEJMoa1200690.

26. Reck M, Rodriguez-Abreu D, Robinson AG, et al; KEYNOTE-024 Investigators. Pembrolizumab versus chemotherapy for PD-L1positive non-small-cell lung cancer. N Engl J Med. 2016 Nov 10;375(19):1823-1833.

27. Ribas A. Releasing the brakes on cancer immunotherapy. N Engl J Med. 2015 Oct 15;373(16):1490-1492. doi: 10.1056/ NEJMp1510079.

28. Larkin J, Chiarion-Sileni V, Gonzalez R, et al. Combined nivolumab and ipilimumab or monotherapy in untreated melanoma. N Engl J Med. 2015 Jul 2;373(1):23-34. doi: 10.1056/ NEJMoa1504030.

29. Armand P, Shipp MA, Ribrag V, et al. Programmed death-1 blockade with pembrolizumab in patients with classical Hodgkin lymphoma after brentuximab vedotin failure. J Clin Oncol. 2016 Nov 1;34(31):3733-3739. doi: 10.1200/ JCO.2016.67.3467.

30. Ansell SM, Lesokhin AM, Borrello I, et al. PD-1 blockade with nivolumab in relapsed or refractory Hodgkin's lymphoma. N Engl J Med. 2015 Jan 22;372(4):311-319. doi: 10.1056/ NEJMoa1411087.

31. Chen R, Zinzani PL, Fanale MA, et al; KEYNOTE-087. Phase II study of the efficacy and safety of pembrolizumab for relapsed/refractory classic Hodgkin lymphoma. J Clin Oncol. 2017 Jul 1;35(19):2125-2132. doi: 10.1200/JCO.2016.72.1316.

32. Lesokhin AM, Ansell SM, Armand P, et al. Nivolumab in patients with relapsed or refractory hematologic malignancy: preliminary results of a phase lb study. J Clin Oncol. 2016 Aug 10;34(23):2698-2704. doi: 10.1200/JCO.2015.65.9789. 
33. Ding W, LaPlant BR, Call TG, et al. Pembrolizumab in patients with CLL and Richter transformation or with relapsed CLL. Blood. 2017 Jun 29;129(26):3419-3427. doi: 10.1182/blood2017-02-765685.

34. Foran AE, Nadel HR, Lee AF, Savage KJ, Deyell RJ. Nivolumab in the treatment of refractory pediatric hodgkin lymphoma. $J$ Pediatr Hematol Oncol. 2017 Jul;39(5):e263-e266. doi: 10.1097/ MPH.0000000000000703.

35. Lussier DM, O'Neill L, Nieves LM, et al. Enhanced T-cell immunity to osteosarcoma through antibody blockade of PD1/PD-L1 interactions. J Immunother. 2015 Apr;38(3):96-106. doi: 10.1097/CJI.0000000000000065.

36. Karim LA, Wang P, de Guzman J, et al. PDL1 protein expression and tumor mutation burden in hematologic malignancies: correlation with Hodgkin and high grade lymphoma. Cancer Res. 2017 Jul;77(13 Suppl):3724.

37. Shi L, Chen S, Yang L, Li Y. The role of PD-1 and PD-L1 in T-cell immune suppression in patients with hematological malignancies. J Hematol Oncol. 2013 Sep 30;6(1):74. doi: 10.1186/1756-8722-6-74.

38. Andorsky DJ, Yamada RE, Said J, Pinkus GS, Betting DJ, Timmerman JM. Programmed death ligand 1 is expressed by non-hodgkin lymphomas and inhibits the activity of tumorassociated T cells. Clin Cancer Res. 2011 Jul 1;17(13):4232-4244. doi: 10.1158/1078-0432.CCR-10-2660.

39. Yamamoto R, Nishikori M, Tashima M, et al. B7-H1 expression is regulated by MEK/ERK signaling pathway in anaplastic large cell lymphoma and Hodgkin lymphoma. Cancer Sci. 2009 Nov;100(11):2093-2100. doi: 10.1111/j.1349-7006.2009.01302.x.

40. Yamamoto R, Nishikori M, Kitawaki T, et al. PD-1-PD-1 ligand interaction contributes to immunosuppressive microenvironment of Hodgkin lymphoma. Blood. 2008 Mar 15;111(6):3220-3224. doi: 10.1182/blood-2007-05-085159.

41. Chen X, Liu S, Wang L, Zhang W, Ji Y, Ma X. Clinical significance of B7-H1 (PD-L1) expression in human acute leukemia. Cancer Biol Ther. 2008 May;7(5):622-627.

42. Yao $Y$, Tao R, Wang X, Wang Y, Mao Y, Zhou LF. B7-H1 is correlated with malignancy-grade gliomas but is not expressed exclusively on tumor stem-like cells. Neuro Oncol. 2009 Dec;11 (6):757-766. doi: 10.1215/15228517-2009-014.

43. Wintterle $S$, Schreiner B, Mitsdoerffer $M$, et al. Expression of the B7-related molecule $\mathrm{B} 7-\mathrm{H} 1$ by glioma cells: a potential mechanism of immune paralysis. Cancer Res. 2003 Nov 1;63 (21):7462-7467.

44. Pinto N, Park JR, Murphy E, et al. Patterns of PD-1, PD-L1, and PD-L2 expression in pediatric solid tumors. Pediatr Blood Cancer. 2017 Nov;64(11). doi: 10.1002/pbc.26613.

45. Lucchesi M, Sardi I, Puppo G, Chella A, Favre C. The dawn of "immune-revolution" in children: early experiences with checkpoint inhibitors in childhood malignancies. Cancer Chemother Pharmacol. 2017 Dec;80(6):1047-1053. doi: 10.1007/ s00280-017-3450-2.
46. Merchant MS, Wright M, Baird K, et al. Phase I clinical trial of ipilimumab in pediatric patients with advanced solid tumors. Clin Cancer Res. 2016 Mar 15;22(6):1364-1370. doi: 10.1158/ 1078-0432.CCR-15-0491.

47. Bajčiová V. Therapeutic effect and tolerance of ipilimumam in metastatic malignant melanoma in children - a case report [in Czech]. Klin Onkol. 2015;28 Suppl 4:4S115-4S120.

48. Shad AT, Huo JS, Darcy C, et al. Tolerance and effectiveness of nivolumab after pediatric T-cell replete, haploidentical, bone marrow transplantation: a case report. Pediatr Blood Cancer. 2017 Mar;64(3). doi: 10.1002/pbc.26257.

49. Bouffet E, Larouche V, Campbell BB, et al. Immune checkpoint inhibition for hypermutant glioblastoma multiforme resulting from germline biallelic mismatch repair deficiency. J Clin Oncol. 2016 Jul 1;34(19):2206-2211. doi: 10.1200/JCO.2016.66.6552.

50. Zhu X, McDowell MM, Newman WC, Mason GE, Greene S, Tamber MS. Severe cerebral edema following nivolumab treatment for pediatric glioblastoma: case report. J Neurosurg Pediatr. 2017 Feb;19(2):249-253. doi: 10.3171/2016.8. PEDS16326.

51. Blumenthal DT, Yalon $M$, Vainer GW, et al. Pembrolizumab: first experience with recurrent primary central nervous system (CNS) tumors. J Neurooncol. 2016 Sep;129(3):453-460.

52. Saha A, Aoyama K, Taylor PA, et al. Host programmed death ligand 1 is dominant over programmed death ligand 2 expression in regulating graft-versus-host disease lethality. Blood. 2013 Oct 24;122(17):3062-3073. doi: 10.1182/blood2013-05-500801.

53. Blazar BR, Carreno BM, Panoskaltsis-Mortari A, et al. Blockade of programmed death-1 engagement accelerates graft-versushost disease lethality by an IFN-gamma-dependent mechanism. J Immunol. 2003 Aug 1;171(3):1272-1277.

54. Haverkos BM, Abbott D, Hamadani M, et al. PD-1 blockade for relapsed lymphoma post-allogeneic hematopoietic cell transplant: high response rate but frequent GVHD. Blood. 2017 Jul 13;130(2):221-228. doi: 10.1182/blood-2017-01-761346.

55. Wolchok JD, Kluger H, Callahan MK, et al. Nivolumab plus ipilimumab in advanced melanoma. N Engl J Med. $2013 \mathrm{Jul}$ 11;369(2):122-133. doi: 10.1056/NEJMoa1302369.

56. Schwab KS, Kristiansen G, Schild HH, Held SEA, Heine A, Brossart $P$. Successful treatment of refractory squamous cell cancer of the head and neck with nivolumab and ipilimumab. Case Rep Oncol. 2018 Jan 4;11(1):17-20. doi: 10.1159/ 000485562.

57. Carter T, Shaw H, Cohn-Brown D, Chester K, Mulholland P. Ipilimumab and bevacizumab in glioblastoma. Clin Oncol $(R$ Coll Radiol). 2016 Oct;28(10):622-626. doi: 10.1016/j. clon.2016.04.042.

58. Hodi FS, Lawrence D, Lezcano $C$, et al. Bevacizumab plus ipilimumab in patients with metastatic melanoma. Cancer Immunol Res. 2014 Jul;2(7):632-642. doi: 10.1158/2326-6066. CIR-14-0053.

This article meets the Accreditation Council for Graduate Medical Education and the American Board of Medical Specialties Maintenance of Certification competencies for Patient Care and Medical Knowledge. 